\title{
QUIMIOTERAPIA DE BANANA ‘PRATA ANÃ' NO CONTROLE DE PODRIDÕES EM PÓS-COLHEITA
}

\author{
W.S. Moraes ${ }^{1}$, L. Zambolim ${ }^{2}$, J.D. Lima ${ }^{3}$
}

${ }^{1}$ Agência Paulista de Tecnologia dos Agronegócios, Pólo do Vale do Ribeira, Av. Wild José de Souza, 454, CEP 11900-000, Registro, SP, Brasil. E-mail: wilson@apta.sp.gov.br

\section{RESUMO}

\begin{abstract}
A antracnose (Colletotrichummusae) e a podridão da coroa (complexo de fungos) são as principais doenças que ocorrem em pós-colheita de bananas. Prolongar a vida pós-colheita, aumentar o período livre dessas doenças e manter a qualidade comestível dos frutos passam a ser o grande desafio. O objetivo do trabalho foi avaliar a eficiência de fungicidas sistêmicos e protetores no controle de podridões em frutos de banana 'Prata anã' proveniente de Jaíba, Minas Gerais. Fungicidas sistêmicos dos grupos químicos dos benzimidazóis, triazóis, imidazóis e piridinamina protetor foram avaliados nas dosagens de $0,125,250$ e $500 \mathrm{~mL} . \mathrm{L}^{-1}$ ou mg.L $\mathrm{L}^{-1}$, após imersão por 3 minutos, sob condições ambientes $\left(18-23^{\circ} \mathrm{C} \mathrm{e} 60-85 \%\right.$ UR). Procloraz $\left(125 \mathrm{~mL}^{-\mathrm{L}^{-1}}\right)$ e propiconazole (250 mL.L $\mathrm{L}^{-1}$ ) foram os mais eficientes em evitar a incidência de podridões em bananas inoculadas com C. musae. Dosagens superiores a estas causaram toxidez aos frutos. Os benzimidazóis não foram eficientes no controle das podridões, mesmo na maior dosagem utilizada (500 mL.L-1 $)$, mostrando-se semelhantes ao fungicida padrão (benomil) e ao controle não-tratado. A eficiência do fungicida propiconazole foi confirmada para uso em pós-colheita da cultura da bananeira, mostrando-se efetivo na dosagem de $250 \mathrm{~mL} . \mathrm{L}^{-1}$, sem causar toxidez em banana 'Prata anã'.
\end{abstract}

PALAVRAS-CHAVE: Controlequímico, antracnose, fungos, podridão da coroa, doenças, fungicida.

\section{ABSTRACT}

CHEMOTHERAPY OF "PRATA ANÃ" BANANAS FOR POST-HARVEST ROT CONTROL. Anthracnose (Colletotrichum musae) and crown rots (fungi complex) are the main diseases that occur in the banana crop post-harvest. This poses the challenge of prolonging the post-harvest life, increasing the disease-free period and maintaining the fruit's food quality. The objective of this work was to evaluate the efficiency of systemic and protective fungicides in the control of postharvest rot in fruits of the banana cultivar "Prata anã" from Jaíba, Minas Gerais, Brazil. Systemic fungicides from the benzimidazole, triazole, imidazole and pyridinamine chemical groups were evaluated in the dosages $0,125,250$ and $500 \mathrm{~mL} . \mathrm{L}^{-1}$ or mg.L $\mathrm{L}^{-1}$, after immersion for 3 minutes, in environmental conditions (18-23 ${ }^{\circ} \mathrm{C}$ and $\left.60-85 \% \mathrm{RU}\right)$. Procloraz $\left(125 \mathrm{~mL} . \mathrm{L}^{-1}\right)$ and propiconazole $\left(250 \mathrm{~mL} . \mathrm{L}^{-1}\right)$ were the most efficient for preventing the incidence of rot in bananas inoculated with C. musae. Higher dosages caused toxicity in the fruits. The benzimidazois were not efficient in the control of rot, even at the highest dosage used $\left(500 \mathrm{~mL} . \mathrm{L}^{-1}\right)$, showing results similar to standard fungicide (benomil) and the control treatment without fungicide. The fungicide propiconazole at the dosage $250 \mathrm{~mL} . \mathrm{L}^{-1}$ proved to be effective for use in the post-harvest bananas, not causing toxicity in the "Prata anã" banana fruits.

KEY WORDS: Chemical control, anthracnose, crown rot, fungi, disease, fungicide.

\section{INTRODUÇÃO}

A antracnose (Colletotrichum musae (Berck. and Curt.) Arx) e a podridão da coroa (complexo de fungos) são as principais doenças que ocorrem em póscolheita de bananas e principais fatores que compro- metem a vida e a qualidade dos frutos nesta fase. Vários outros patógenos podem estar associados a essas podridões, como Chalara paradoxa (Desynes) Sacc., Botryodiplodia theobromae Pat. e Verticillium theobromae (Turconi) Mason and Hughes. (Alves etal., 1995).

\footnotetext{
${ }^{2}$ Universidade Federal de Viçosa, Departamento de Fitopatologia, Lavras, MG, Brasil.

${ }^{3}$ Universidade Estadual Paulista, Registro, SP, Brasil.
} 
No entanto, prolongar a vida pós-colheita e aumentar o período livre dessas doenças, sem alterar a qualidade comestível dos frutos, torna-se um grande desafio. Atualmente, as tecnologias disponíveis e mais praticadas no mundo são a quimioterapia e a refrigeração (JOHANSON; SANGCHOTE, 1994).

A principal forma de controle das doenças de frutos em pós-colheita continua sendo os fungicidas, porém, sua forma de aplicação (WISNIEWSKI; WILSON, 1992), o surgimento de raças de patógenos resistentes (JONES, 1991; Ventura; BALBINO, 1994; FERNANDEs et al., 1994; LAPEYRE De BALLAIRE;Dubois, 1997) e as pressões socioeconômicas têm reduzido as oportunidades de se planejar estratégias de controle baseadas em fungicidas.

Desenvolvidos desde 1965, os benzimidazóis atuam nos processos de divisão celular como inibidores da mitose, e os triazóis como inibidores da biossíntese de esteróis. Assim, são os mais recomendados para controle de antracnoses e podridões em frutos cítricos, banana, mamão, manga e abacate (ECKERT,1990). Estes fungicidas apresentam vantagens de atuarem em amplo espectro de doenças causadas por deuteromicetes, basidiomicetes e ascomicetes, exceto os oomicetes que não sintetizam esteróis. No entanto, a perda da eficiência desses e de outros fungicidas aplicados em pré-colheita ameaça sua utilização em pós-colheita.

Ouso intensivo desses fungicidas em pré-colheita tem levado ao desenvolvimento de raças de patógenos tolerantes a esses produtos. Raças de Colletotrichum tolerantes aos benzimidazóis foram relatadas em mangas e bananas (ECKERT, 1990); JOHANSON; BLAZQUEZ (1992). Apesar dos baixos riscos de adaptabilidade, biótipos tolerantes a imazalil foram encontrados na Califórnia, porém não detectados na Flórida. Fungos como Acremonium strictum, Alternaria sp., Cladosporium sp., Curvularia brachyspora, Pestalotiopsis cruenta,Phoma joliana Verticillium theobromae mostraram-se tolerantes a benomil, thiabendazole e carbendazim (Mesturino,1988).

O benomil e o tiabendazole são os fungicidas mais recomendados para controle de doenças em pré e pós-colheita de mamão e bananas. No entanto, têm-se mostrado menos efetivos que o procloraz e o propiconazole (SePIAH, 1994). Enquanto benzimidazóis mostraram-se ineficientes no controle de Colletotrichum em mamão e banana, in vitro procloraz e fluazinan foram os mais efetivos, mesmo na concentração de $1 \mathrm{mg} \cdot \mathrm{mL}^{-1}$ (VENTURA;B ALBINO, 1994).

A eficiência desses produtos no controle das doenças depende da quantidade do inóculo inicial, da profundidade da infecção no hospedeiro, da taxa de crescimento da infecção, da temperatura e umidade e do grau de penetração do produto no tecido hospedeiro (Tongthieng; SAngchote, 1994; Ploetz et al., 1994).

No Brasil, vários fungicidas têm sido recomendados no tratamento pós-colheita de bananas incluindo mancozeb, benomil, thiabendazoleetiofanato metílico (Alves et al., 1995). Em outros países, além destes, são indicados o prochloraz e imazalil (JONES, 1991; PLOETZ et al., 1994; Tongthieng; SANGchote, 1994).

Diferentes respostas foram obtidas dentro de uma mesma cultivar em diferentes locais. Na Austrália, por exemplo, bananas 'Cavendish' apresentaram melhor controle da antracnose quando tratadas com imazalil a 500 mg. L $^{-1}$ (Tongthieng; SANGchote, 1994), enquanto no Brasil, Cordeiro etal. (1996) apontam o prochloraz e o propiconazole (100 mg. $\left.\mathrm{L}^{-1}\right)$ como os mais eficientes no controle da doença nessa cultivar.

A escolha de um fungicida pode ser influenciada pela interação fruto-patógeno-fungicida, pelo registro do produto para a cultura e pela exigência do país importador. As normas e padrões de qualidade de banana para exportação incluem os níveis residuais de produtos, remanescentes na polpa, utilizados no controle de pragas e doenças e são definidos pelos próprios importadores. Os Estados Unidos e a Europa, por exemplo, permitem que os frutos sejam tratados com thiabendazole e imazalil (Jones; Slabaugh, 1994). Na Europa, enquanto alguns parlamentares têm articulado a proibição do uso de pesticidas em frutos e hortaliças, outros preferem definir limites de tolerância e efeitos residuais dos produtos.

Diante do exposto, este estudo teve por objetivo avaliar a eficiência de fungicidas sistêmicos e protetores no controle de podridões em fruto de banana 'Prata anã' proveniente do norte do Estado de Minas Gerais.

\section{MATERIAL E MÉTODOS}

O experimento foi conduzido nos Laboratórios de Pós-colheita e de Fitopatologia da Universidade Federal de Viçosa, em Viçosa, Minas Gerais, nos meses de maio e junho de 1998. Para tal, cachos de banana 'Prata anã' foram colhidos em estádio préclimatérico de plantações comerciais de dois anos de idade, no Município de Jaíba, MG. Em seguida, foram despencados e as pencas selecionadasforam acondicionadas em caixas plásticas e transportadas por caminhão, em doze horas, até a Universidade Federal de Viçosa. Na seleção, optou-se pelas pencas centrais dos cachos, rejeitando-se as duas pencas proximais e as três pencas distais, visando maior uniformidade dos frutos durante a maturação em pós-colheita. 
Já no Laboratório de Pós-colheita daUFV, buquês, contendo cinco frutos, foram retirados dos cachos e atomizados com uma suspensão de 2,5.106 esporos. $\mathrm{mL}^{-1}$ de C. musae, obtida de colônias monospóricas cultivadas em BDA, durante sete dias. Após a inoculação, os buquês foram incubados sob câmara úmida a $25^{\circ} \mathrm{C}$, por 24 horas.

Decorrido esse período, os buquês foram imersos em diferentes suspensões defungicidas. Osfungicidas sistêmicos selecionados pertencem ao grupo químico dos benzimidazóis: benomil, tiabendazole, carbendazim; triazóis: propiconazole; imidazóis: procloraz e piridinamina: fluazinan, nas dosagens de $0,125,250$ e $500 \mathrm{mg}$. $\mathrm{L}^{-1}$ por 3 minutos. Oito buquês foram tratados, individualmente, em recipiente plástico com 20 L de suspensão fungicida, suplementada com 2,0 mL do adjuvante triton-100.

Decorrido o tempo de imersão, os buquês foram retirados, deixados secar e colocados em caixas plásticas forradas com fina camada de papel picado. As caixas foram distribuídas no laboratório, de acordo com o delineamento experimental e mantidas, sob condição ambiente $\left(18-23^{\circ} \mathrm{C}\right.$ e $60-85 \%$ UR), até o completo amadurecimento.

Os experimentos foram dispostos em parcelas subdivididas, tendo nas parcelas um esquema fatorial $6 \times 4$ (6 fungicidas e 4 doses) e, nas subparcelas, o número de épocas de avaliação, em delineamento inteiramente casualizado com três repetições, de quatro buquês cada.

A incidência de podridões, a percentagem da área lesionada por fruto e a evolução da cor dos buquês foram avaliadas a 0,3, 6, 9 e 12 dias após os tratamen- tos. A incidência foi determinada pelo temponecessário para o início de aparecimento dos sintomas e a percentagem da área lesionada por fruto determinada com auxílio de uma escala diagramática de 0 a $64 \%$ da área lesionada do fruto, desenvolvida exclusivamente para uso neste estudo(Fig. 1). A evolução da cor dos buquês foi determinada com auxílio da escala visual apresentada por DADZIE; ORCHARD (1996), com índices de cor variando de 1 a 7 , sendo que 1 equivale ao fruto completamente verde e, 7 ao fruto totalmente amarelo com manchas castanhas.

Para a avaliação da eficiência de fungicidas sistêmicos e protetores no controle de podridões em frutos de banana 'Prata anã' utilizou-se o benomil como o fungicida padrão para comparação.

Ao término do período decarência foram coletadas amostras da casca e da polpa dos frutos para análise de resíduos dos fungicidas propiconazole na dose de $250 \mathrm{~mL} . \mathrm{L}^{-1}$ que foram realizadas no Instituto Mineiro de Agropecuária.

Quando o teste F foi significativo na análise de variância, as médias dos tratamentos foram comparadas pelo teste Tukey a 5\% de probabilidade.

\section{RESULTADOS E DISCUSSÃO}

Os resultados mostraram que os fungicidas do grupo químico dos benzimidazóis, como benomil, thiabendazole e carbendazim, mesmo na maior dosagem utilizada (500 mL.L ${ }^{-1}$ ), não foram eficientes no controle das podridões, e mostraram-se semelhantes ao benomil (fungicida padrão) (Fig. 2).

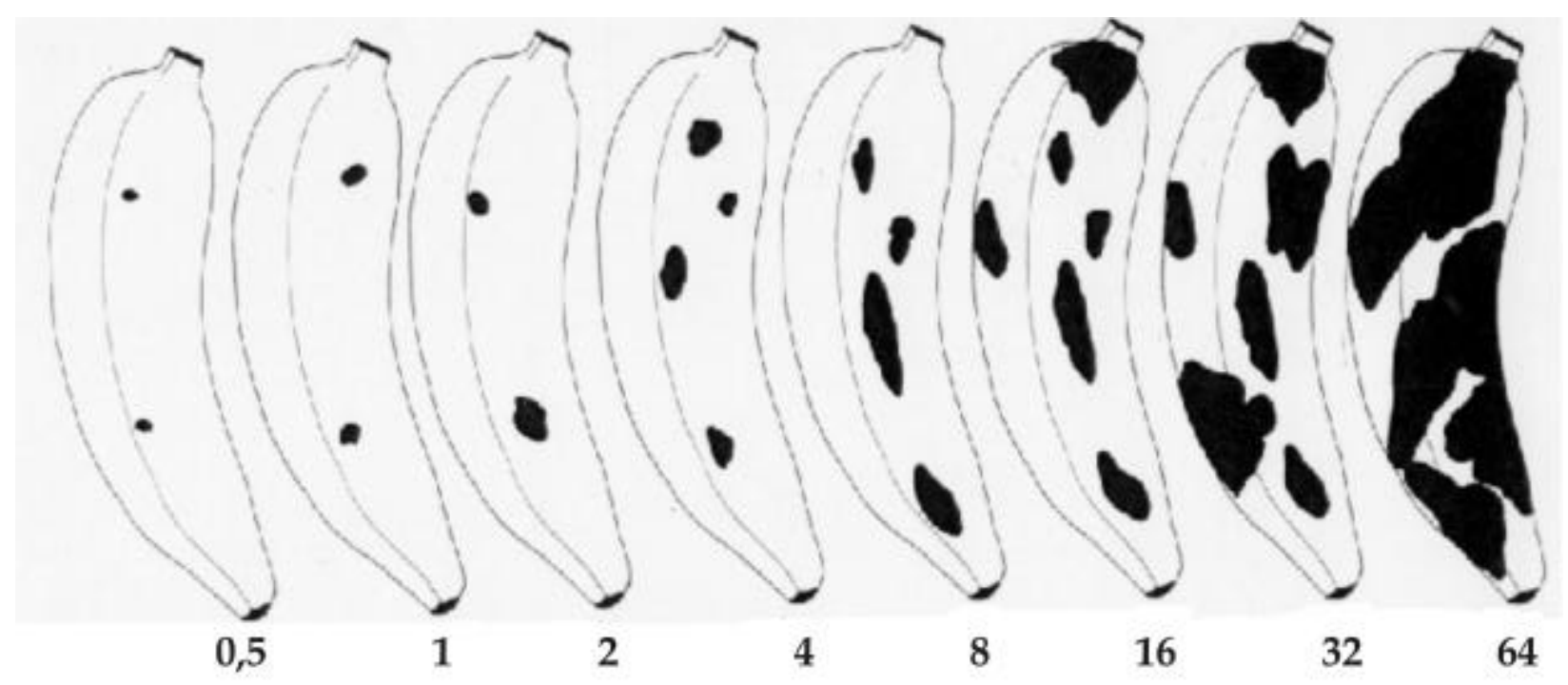

Fig. 1 - Escala diagramática para avaliar a severidade de podridões em frutos de banana 'Prata anã' (AAB), cujos valores correspondem à percentagem de área lesionada/fruto. 

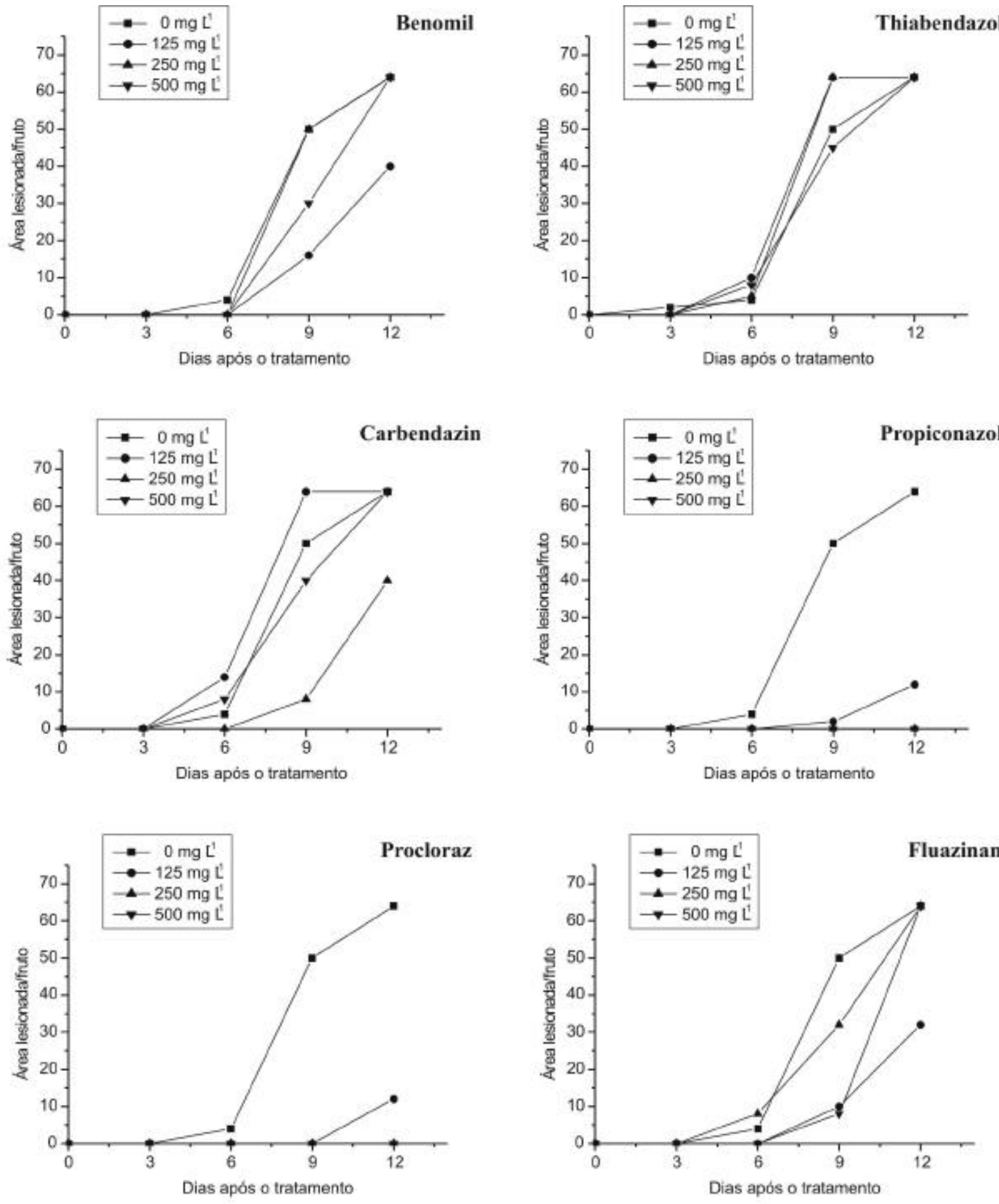

Fig. 2 - Evolução da percentagem de área lesionada/fruto de bananas 'Prata anã', submetidas aos tratamentos com diferentes suspensões fungicidas e dosagens, sob condições ambientes. Valores representam médias provenientes de 3 buquês e 3 repetições por tratamento.

Esses resultados podem ser um indicativo da presença de patógenos tolerantes a estes fungicidas que, apesar de recomendados para controle das doenças em pós-colheita de bananas (ECKERT, 1990; Alves etal., 1995), não se mostraram eficientes. A perda da eficiência pode ser devido ao uso intensivo desses fungicidas, os quais são aplicados em pulverizações aéreas e terrestres para o controle da Sigatoka Amarela em pré-colheita. Esse fato pode ter contribuído para o surgimento de patógenos tolerantes, requerendo, portanto, estudos mais detalhados para confirmação, de acordo com relatos feitos porJones (1991), VentuRA; 
Balbino (1994), Fernandes et al. (1994) e Lapeyre de Ballaire; Dubois (1997).

Os fungicidas procloraz (125 mL. $\left.\mathrm{L}^{-1}\right)$ e propiconazole ( $\left.250 \mathrm{~mL} . \mathrm{L}^{-1}\right)$ foram os mais eficientes no controle das podridões, em frutos de banana inoculados (Fig. 2). A eficiência destes fungicidas pode ser atribuída ao modo de ação que, diferentemente dos benzimidazóis e do fungicida protetor, atuam como inibidores da biossíntese de esteróis. Resultados semelhantes foram obtidos por VENTURA;B ALBINO (1994) e SEPIAH (1994).

A eficiência dos fungicidas em erradicar as infecções depende do tempo entre a infecção e a aplicação do fungicida, do local da estrutura de infecção quiescente e da propriedade de cobertura e penetração da formulação fungicida (HIFFMANN-NADEL; COHEN, 1966; JoHANSON; SANGCHOTE, 1994). Nesse estudo, procloraz e propiconazole foram capazes de erradicar as infecções induzidas artificialmente e as naturais provenientes do campo, independente do tempo da infecção.

Em resultados obtidos em experimentos preliminares confirmaram o fungo $C$. musae como agente primário das podridões em frutos de banana utilizados neste estudo. Entretanto, apenas propiconazole apresenta registro para ser usado na cultura da banana, porém em pré-colheita. Este fungicida sistêmico pertence ao grupo químico dos triazóis, classe toxicológica III, apresenta período de carência de apenas 1 (um) dia e nível de tolerância de $0,1 \mathrm{mg}$.L-1 ou $0,4 \mathrm{mg} \cdot \mathrm{kg}^{-1}$ para bananas.

Procloraz mostrou-se tão efetivo quanto o propiconazole na dosagem de $125 \mathrm{~mL}$. $\mathrm{L}^{-1}$, porém causou sintomas de toxidez na casca dos frutos, cujo tempo de manifestação e a intensidade foram inversa e diretamente proporcionais a dosagem testada, respectivamente. Apesar de poucos sintomas terem sido visualizados nessa dosagem, foi suficiente para comprometer a qualidade externa dos frutos.

Propiconazole mostrou-se efetivo na dosagem de 250 mL.L ${ }^{-1}$, acima da qual verificaram-se sintomas de toxidez e abaixo da qual não se obteve controle de podridões. Os sintomas de toxidez foram caracterizados por um escurecimento na casca dos frutos, especialmente nos locais de injúrias mecânicas, provocadas pela embalagem durante o transporte, ou ainda por pequenas e numerosas manchas escuras em todo o fruto.

Nesse estudo observou-se que os frutos tratados com procloraz (125 mL.L $\left.{ }^{-1}\right)$ e propiconazole $\left(250{\mathrm{~mL} . \mathrm{L}^{-1}}^{-1}\right)$ não apresentaram podridões e se mantiveram com índice de cor 5 , sendo que os frutos tratados com benomil (500 mL.L-1 $)$ e os não-tratados apresentaram $50 \%$ e $64 \%$ da área lesionada/fruto, respectivamente, eíndice de cor 6, aos 12 dias após os tratamentos. Essa pequena diferença na percentagem de podridões, apesar de não significativa, representa o efeito das doenças sobre a deterioração dos frutos. Apesar de recomendado para o controle da Sigatoka Amarela e Negra na cultura da bananeira, o propiconazole também mostrou-se altamente eficiente no controle de podridões em pós-colheita.

A análise de resíduos em frutos que receberam propiconazole por meio de cromatografia realizada no Instituto Mineiro de Agropecuária demonstrou a presença de resíduo do fungicida abaixo do nível tolerável, um dia após sua aplicação, confirmando a degradação desse fungicida no fruto de banana.

Diante da eficiência do fungicida propiconazole, recomenda-se a realização de novos estudos com este fungicida no sentido de validar a concessão de registro para nova utilização em pós-colheita de banana, seguindo as normas do Ministério da Agricultura, Pecuária e Abastecimento.

\section{REFERÊNCIAS}

ALVES, E.J.; DANTAS, J.L.L.; SOARES FILHO, W.S. Banana para exportação: aspectos técnicos da produção. Brasília: Embrapa, 1995. 106p. (Série Publicações Técnicas: FRUPEX, 18).

CORDEIRO, Z.J.M.; PIMENTEL, J.P.; GÓES, A. de; KIMATI, H. Controle químico in vitro e in vivo da antracnose de banana em pós-colheita. Fitopatologia Brasileira, v.21, p.397, 1996. Suplemento 1.

DADZIE, B.K.; ORCHARD, J.E. Post-harvest criteria and methods for routine screening of banana/plantain hybrids. In: INTERNATIONAL NETWORK FOR

IMPROVMENT OF BANANA AND PLANTAIN

(INIBAP), 1966. Montpellier. Proceedings. Montpellier, 1996. 64p

ECKERT, A. Recent developments in the chemical control of postharvest diseases. Acta Horticulturae, v.269, p.477-494, 1990.

FERNANDES, C.A.; RIBEIRO, L.D.; SANTOS, A.S.; AKIBA, F. Ocorrência de isolados de Colletotrichum gloeosporioides resistentes ao fungicida benomil. Fitopatologia Brasileira, v.19, p.284, 1994. Suplemento 1.

JOHANSON, G.I.; SANGCHOTE, S. Control of postharvest disease of tropical fruits: challenges for the 21st century. In: CHAMP, B.R.; HIGHLEY; E.; JOHANSON, G.I. (Ed.). Postharvest handling of tropical fruits. Camberra: ACIAR, 1994. p.140-161.

JOHANSON, A.; BLAZQUEZ, B. Fungi associated with banana crown rot on field-packed fruit from the Windward Islands and assessment of their sensitivity to the fungicides thiabendazole, prochloraz and imazalil Crop Protection, v.11, n.1, p.79-83, 1992 
JONES, D.R. Chemical control of crown rot in Queensland bananas. Australian Journal of Experimental Agriculture, v.31, n.5, p.693-698, 1991.

JONES, D.R.; SLAUBAUGH, W.R. Banana disease caused by fungi: antracnose and fungal scald. In: PLOETZ, R.C.; ZENTMYER, W.T.; NISHIGIMA, K.G.; ROHRBACK, H.D. (Ed.). Compendium of tropical fruits disease. Minessota: APS Press, 1994. p.4-5.

LAPEYRE DE BELLAIRE, L. de; DUBOIS, C. Distribution of thiabendazole-resistant Colletotrichum musae isolates from Guadeloupe banana plantations. Plant Disease, v.81, n.12, p.1378-1383, 1997.

MESTURINO, L. Disease of banana fruits: evaluation of alternative fungicides. Rivista di Agricoltura Subtropicale e Tropicale, v.82, p.517-524, 1988.

PLOETZ, R.C.; ZENTMYER, G.A; NISHIJIMA, W.T.; ROHRBACH, K.G.; OHR, H.D. Compendium of tropical fruits disease. Minnesota: APS Press, 1994. 88p.

SEPIAH, M. Efficacy of propiconazole against fungi causing post-harvest disease on Eksotica papaya. In: CHAMP, B.R.; HIGHLEY; E.; JOHANSON, G.I. (Ed.). Postharvest handling of tropical fruits. Camberra: ACIAR, 1994. p.455-457.
SHIFFMANN-NADEL, M.; COHEN, E. Influence of growth temperatures on the effectiveness of heat treatment of Phytophthora infected citrus fruits. Plant Disease Reporter, v.50, p.867-868, 1966.

TONGTHIENG, T.; SANGCHOTE, S. Postharvest fruit rot banana caused by Colletotrichum musae (Berk y Curt.) Arx. and its control. In: CHAMP, B.R.; HIGHLEY; E.; JOHANSON, G.I. (Ed.). Postharvest handling of tropical fruits. Camberra: ACIAR, 1994. p.462463.

VENTURA, J.A; BALBINO, J.M. de S. Resistência do agente etiológico da antracnose do mamoeiro ao benomil, no estado do Espírito Santo. Fitopatologia Brasileira, v.19, p.297, 1994. Suplemento 1.

WISNIEWSKI, M.E.; WILSON, C.L. Biological control of post-harvest disease of fruits and vegetables: Recent Advances. Hortscience, v.27, n.22, p.94-98, 1992.

Recebido em 10/7/06

Aceito em 5/3/08 